\title{
Subsequent opioid overdose resulting in death in individuals in Allen County, Indiana who receive naloxone administration for past opioid overdose \\ Cassandra Schwartz ${ }^{1}$, Thomas Dykstra ${ }^{2}$, Jana Sanders ${ }^{3}$ \\ Indiana University School of Medicine ${ }^{1}$, Parkview Health ${ }^{2}$, Allen County Department of Health ${ }^{3}$
}

\section{Background and Hypothesis:}

Over-prescription of opioids has contributed to increases in opioid overdose (OOD) and OOD deaths. Studies suggest recurring OODs in an individual could be contributing. After a non-fatal overdose, one study found $7 \%$ of patients continued to abuse opioids and had another OOD within 1 year. Additionally, the literature suggests a positive correlation between non-fatal overdose and increased risk of subsequent overdose death. Allen County has also experienced increases in overdoses and overdose deaths. We aim to isolate opioid-specific overdoses and deaths in Allen County from current overdose data. We hypothesize individuals in Allen County who received naloxone for OOD have an increased risk of death from a subsequent OOD. An additional objective is to utilize data from the county ambulance service and the two major Allen County health systems to gain more accurate and current accountings of the OOD data.

\section{Methods:}

This study is a retrospective case-control using electronic medical records and/or electronic patient care reports from the Three Rivers Ambulance Authority, Parkview Health, and Lutheran Health Network. Patients who received naloxone between 2018-2020 will be divided into a case group of individuals who died of OOD and a control group of living individuals. Demographics and naloxone administrations data per subject will be collected. Box-and-whisker plots, a MannWhitney U Test, and a Wilcoxon matched-pairs signed rank test will be done for comparisons.

\section{Results and Conclusion:}

This project has resulted in a study protocol ready for IRB submission with IRB approval and study start date anticipated for Summer 2021.

\section{Potential Impact:}

This study will add to existing knowledge on OOD and subsequent OOD death. Specifically, this study will provide more accurate and current accountings of the OOD data that may aid in the development of policy and interventions to reduce the local opioid crisis. 